DOI: $10.15393 /$ j3.art.2021.9230

UDC 517.988, 519.6, 531

G. Argyros, M. Argyros, I. K. Argyros, S. George

\title{
SEMI-LOCAL CONVERGENCE OF A DERIVATIVE-FREE METHOD FOR SOLVING EQUATIONS
}

\begin{abstract}
We present the semi-local convergence analysis of a two-step derivative-free method for solving Banach space valued equations. The convergence criteria are based only on the first derivative and our idea of recurrent functions.
\end{abstract}

Key words: Banach space, derivative-free method, semi-local convergence

2010 Mathematical Subject Classification: 65G99, 65J20, 49M15, 74G20, 41A25

1. Introduction. Let $B_{1}, B_{2}$ stand for Banach spaces, $U(x, \rho)$ denote a closed ball with center $x \in B_{1}$ and of radius $\rho>0$. We denote the closure of $U(x, \rho)$ by $\bar{U}(x, \rho)$.

We are dealing with the problem of approximating a solution $x_{*}$ of equation

$$
F(x)=0 .
$$

Solving equation (1) is very important, because many problems are reduced to it by Mathematical modeling [1-8]. The solution methods are usually of iterative nature, since solutions in the closed form are rarely obtained. In this article, we develop a derivative-free method to generate a sequence approximating $x_{*}$ under certain conditions. The method is defined for all $n \geqslant 0$ as

$$
\begin{aligned}
y_{n} & =x_{n}-B_{n}^{-1} F\left(x_{n}\right) \\
x_{n+1} & =x_{n}-A_{n}^{-1} F\left(x_{n}\right),
\end{aligned}
$$

where $A_{n}=\left[\frac{x_{n}+y_{n}}{2}, x_{n} ; F\right](n \geqslant 0), B_{n}=\left[\frac{x_{n-1}+y_{n-1}}{2}, x_{n} ; F\right](n \geqslant 1)$, and $x_{0}, y_{0} \in \Omega$ are initial points. Here, $[x, y ; F]: \Omega \times \Omega \longrightarrow L(X, Y)$ denotes a divided difference of order one for the operator $F$ at the point

(C) Petrozavodsk State University, 2021 
$x, y \in D$ (see [2], [6], [8]). The method (2) is a useful alternative to thirdorder methods, such as the method of tangent hyperbolas (Halley) or the method of tangent parabolas (Euler-Chebysheff) [1-8]. However, these methods are very expensive, since they require the evaluation of the second Fréchet derivative at each step. Discretized versions of these methods, such as Ulm's method, use divided differences of order one [1-8].

The rest of the paper is organized as follows. In Section 2, we present the semi-local convergence analysis of method (2), whereas in the concluding Section 3, we present the numerical examples.

2. Semi-local convergence. Semi-local convergence is based on the majorant sequence defined for $n=1,2, \ldots$ and some $\eta \geqslant 0$ and $s \geqslant 0$, as follows:

$$
\begin{aligned}
t_{0} & =0, \quad t_{1}=\eta \geqslant 0, \quad s_{0}=s \geqslant 0 \\
s_{n} & =t_{n}+\frac{L\left(2\left(t_{n}-t_{n-1}\right)+\left(s_{n-1}-t_{n-1}\right)\right)\left(t_{n}-t_{n-1}\right)}{2\left[1-\frac{L_{0}}{2}\left(t_{n-1}+s_{n-1}+2 t_{n}+s\right)\right]} \\
t_{n+1} & =t_{n}+\frac{L\left(2\left(t_{n}-t_{n-1}\right)+\left(s_{n-1}-t_{n-1}\right)\right)\left(t_{n}-t_{n-1}\right)}{2\left[1-\frac{L_{0}}{2}\left(3 t_{n}+s_{n}+s\right)\right]} .
\end{aligned}
$$

Define the scalar cubic polynomial $p$ as

$$
p(t)=L_{0} t^{3}+3 L_{0} t^{2}+3 L t-3 L \text { for some } L_{0}>0 \text { and } L>0 .
$$

By this definition, $p(0)=-3 L$ and $p(1)=4 L_{0}$. It follows from the intermediate value theorem and the Descartes rule of signs, that polynomial $p$ has a unique root $\gamma \in(0,1)$. Moreover, define $\alpha_{0}$ and $\alpha_{1}$ as

$$
\begin{gathered}
\alpha_{0}=\frac{L\left(2\left(t_{1}-t_{0}\right)+s_{0}-t_{0}\right)}{2\left[1-\frac{L_{0}}{2}\left(3 t_{1}+s_{1}+s\right)\right]}, \\
\alpha_{1}=\frac{L\left(2\left(t_{1}-t_{0}\right)+s_{0}-t_{0}\right)}{2\left[1-\frac{L_{0}}{2}\left(t_{0}+s_{0}+2 t_{1}+s\right)\right]},
\end{gathered}
$$

and set

$$
\gamma_{0}=\max \left\{\alpha_{0}, \alpha_{1}\right\}
$$

Next, we present a convergence result for the majorizing sequence $\left\{t_{n}\right\}$.

Lemma 1. Suppose that there exists $\gamma$, such that $L_{0} s<2$ and

$$
0<\gamma_{0} \leqslant \gamma \leqslant 1-\frac{L_{0} \eta}{1-\frac{L_{0}}{2} s} \text {. }
$$


Then, the sequence $\left\{t_{n}\right\}$ given by (3) is nondecreasing, bounded from above by $t_{* *}=\frac{\eta}{1-\gamma}$, and converges to its unique least upper bounds $t_{*}$, which satisfies $\eta \leqslant t_{*} \leqslant t_{* *}$.

Proof. We shall show, using induction, that

$$
0<\frac{L\left(2\left(t_{k+1}-t_{k}\right)+\left(s_{k}-t_{k}\right)\right.}{2\left[1-\frac{L_{0}}{2}\left(3 t_{k+1}+s_{k+1}+s\right)\right]} \leqslant \gamma
$$

and

$$
0<\frac{L\left(2\left(t_{k+1}-t_{k}\right)+\left(s_{k}-t_{k}\right)\right.}{2\left[1-\frac{L_{0}}{2}\left(t_{k}+s_{k}+2 t_{k+1}+s\right)\right]} \leqslant \gamma .
$$

Estimates (9) and (10) hold for $k=0$ by (3), (5)-(8). Suppose that (9) and (10) hold for $j=1,2, \ldots, k-1$. Then, by (3), (9) and (10),

$$
\begin{gathered}
0<t_{k+1}-t_{k} \leqslant \gamma\left(t_{k}-t_{k-1}\right) \leqslant \gamma^{k} \eta \Longrightarrow t_{k+1} \leqslant \frac{1-\gamma^{k+1}}{1-\gamma} \eta \\
0<s_{k}-t_{k} \leqslant \gamma\left(t_{k}-t_{k-1}\right) \leqslant \gamma^{k} \eta \Longrightarrow s_{k} \leqslant \frac{1-\gamma^{k}}{1-\gamma} \eta+\gamma^{k} \eta=\frac{1-\gamma^{k+1}}{1-\gamma} \eta .
\end{gathered}
$$

By (9) and (10), we must only complete the induction for (9). Evidently, this is true by (3), (11), and (12), provided that

$$
\frac{L}{2}\left(2 \gamma^{k} \eta+\gamma^{k} \eta\right)+\frac{\gamma L_{0}}{2}\left(3 \frac{1-\gamma^{k+1}}{1-\gamma} \eta+\frac{1-\gamma^{k+2}}{1-\gamma} \eta+s\right)-\gamma \leqslant 0 .
$$

Estimate (13) suggests to introduce functions $\varphi_{k}$ on $[0,1)$ as

$$
\varphi_{k}(t)=\frac{3 L}{2} t^{k-1} \eta+\frac{L_{0}}{2}\left[3\left(1+t+\ldots+t^{k}\right)+\left(1+t+\ldots+t^{k+1}\right)\right] \eta .
$$

We seek for a relationship between two consecutive functions $\varphi_{k}$. We can write, in turn,

$$
\begin{aligned}
& \varphi_{k+1}(t)= \\
& =\frac{3 L}{2} t^{k} \eta+\frac{L_{0}}{2}\left(3\left(1+t+\ldots+t^{k+1}\right)+\left(1+t+\ldots+t^{k+2}\right)\right) \eta+\frac{L_{0}}{2} s-1- \\
& -\frac{3}{2} L t^{k-1} \eta-\frac{L_{0}}{2}\left(3\left(1+t+\ldots+t^{k}\right)+\left(1+t+\ldots+t^{k+1}\right)\right) \eta-\frac{L_{0}}{2} s+1+\varphi_{k}(t)= \\
& =\varphi_{k}(t)+p(t) \frac{t^{k-1} \eta}{2},
\end{aligned}
$$


where $p(t)$ is given by (4). In particular, we get

$$
\varphi_{k+1}(\gamma)=\varphi_{k}(\gamma)
$$

by the definition of $\gamma$. Therefore, (13) holds, provided that

$$
\varphi_{\infty}(\gamma) \leqslant 0
$$

where

$$
\varphi_{\infty}(\gamma)=\lim _{k \longrightarrow \infty} \varphi_{k}(\gamma)
$$

However,

$$
\varphi_{\infty}(\gamma)=\frac{2 L_{0} \eta}{1-\gamma}+\frac{L_{0}}{2} s-1
$$

by (13). Hence, (17) holds if

$$
\frac{2 L_{0} \eta}{1-\gamma}+\frac{L_{0}}{2} s-1 \leqslant 0
$$

or

$$
\gamma \leqslant 1-\frac{L_{0} \eta}{1-\frac{L_{0}}{2} s},
$$

which is true, by (8). Then sequence $\left\{t_{n}\right\}$ is nondecreasing and, in view of (11), is bounded from above by $t_{* *}$. Hence, it converges to its unique least upper bound that satisfies $\eta \leqslant t_{*} \leqslant t_{* *}$.

Next, we present the semi-local convergence for the method (2).

Theorem 1. Assume the following:

(i) $F: \Omega \subset B_{1} \longrightarrow B_{2}$ is a continuous operator with a standard divided difference of order one, such that $[\cdot, \cdot]: \Omega \times \Omega \longrightarrow L\left(B_{1}, B_{2}\right)$ and $x_{0}, y_{0} \in \Omega$ are such that $A_{0}=\left[\frac{x_{0}+y_{0}}{2}, x_{0} ; F\right]$ is invertible. Let $\left\|x_{1}-x_{0}\right\| \leqslant \eta$ and $\left\|y_{0}-x_{0}\right\| \leqslant s$.

(ii) Assumptions of Lemma 1 hold.

(iii) $\left\|A_{0}^{-1}\left([x, y ; F]-A_{0}\right)\right\| \leqslant L_{0}\left(\left\|x-\frac{x_{0}+y_{0}}{2}\right\|+\left\|y-x_{0}\right\|\right)$ for all $x, y \in \Omega$ and some $L_{0}>0$. Set $\rho=\frac{1}{4}\left(\frac{2}{L_{0}}-s\right)$ and $\Omega_{0}=\Omega \cap U\left(x_{0}, \rho\right)$.

(iv) $\left\|A_{0}^{-1}([x, y ; F]-[z, y ; F])\right\| \leqslant L\|x-z\|$ for all $x, y, z \in \Omega_{0}$ and some $L>0$.

(v) $\bar{U}\left(x_{0}, t_{*}\right) \subseteq \Omega$. 
Then there exists a limit point $x_{*} \in \bar{U}\left(x_{0}, t_{*}\right)$ of the sequence $\left\{x_{n}\right\}$, such that $F\left(x_{*}\right)=0$.

Proof. We use mathematical induction to show the estimates

$$
\left\|x_{n+1}-x_{n}\right\| \leqslant t_{n+1}-t_{n}
$$

and

$$
\left\|y_{n}-x_{n}\right\| \leqslant s_{n}-t_{n}
$$

These estimates are true due to the initial conditions and (3) for $n=0$. Suppose that the initial conditions and (3) hold for $n=0$. Also suppose that they are true for all $k=0,1,2, \ldots n-1$. Then we have, by (iii):

$$
\begin{gathered}
\left\|A_{0}^{-1}\left(B_{k}-A_{0}\right)\right\| \leqslant L_{0}\left(\left\|\frac{x_{k-1}+y_{k-1}}{2}-\frac{x_{0}+y_{0}}{2}\right\|+\left\|x_{k}-x_{0}\right\|\right) \leqslant \\
\leqslant \frac{L_{0}}{2}\left(\left\|x_{k-1}-x_{0}\right\|+\left\|y_{k-1}-y_{0}\right\|+2\left\|x_{k}-x_{0}\right\|\right) \leqslant \\
\leqslant \frac{L_{0}}{2}\left(2\left\|x_{k-1}-x_{0}\right\|+\left\|y_{k-1}-x_{k-1}\right\|+\left\|y_{0}-x_{0}\right\|+2\left\|x_{k}-x_{0}\right\| \leqslant\right. \\
\leqslant \frac{L_{0}}{2}\left(2\left(t_{k-1}-t_{0}\right)+s_{k-1}-t_{k-1}+2\left(t_{k}-t_{0}\right)+s\right)= \\
=\frac{L_{0}}{2}\left(t_{k-1}+s_{k-1}+2 t_{k}+s\right)<1,
\end{gathered}
$$

which, together with Banach's Lemma on invertible operators, show that $B_{k-1}$ is invertible and

$$
\left\|B_{k}^{-1} A_{0}\right\| \leqslant \frac{1}{1-\frac{L_{0}}{2}\left(t_{k-1}+s_{k-1}+2 t_{k}+s\right)} .
$$

Similarly,

$$
\begin{gathered}
\left\|A_{0}^{-1}\left(A_{k}-A_{0}\right)\right\| \leqslant L_{0}\left(\left\|\frac{x_{k}+y_{k}}{2}-\frac{x_{0}+y_{0}}{2}\right\|+\left\|x_{k}-x_{0}\right\|\right) \leqslant \\
\leqslant \frac{L_{0}}{2}\left(\left\|x_{k}+y_{k}-\left(x_{0}+y_{0}\right)\right\|+2\left\|x_{k}-x_{0}\right\|\right) \leqslant \\
\leqslant \frac{L_{0}}{2}\left(\left\|x_{k}-x_{0}\right\|+\left\|y_{k}-y_{0}\right\|+2\left\|x_{k}-x_{0}\right\|\right) \leqslant \\
\leqslant \frac{L_{0}}{2}\left(3\left\|x_{k}-x_{0}\right\|+\left\|y_{k}-y_{0}\right\|\right) \leqslant \\
\leqslant \frac{L_{0}}{2}\left(3\left\|x_{k}-x_{0}\right\|+\left\|y_{k}-x_{k}\right\|+\left\|x_{k}-x_{0}\right\|+\left\|x_{0}-y_{0}\right\|\right) \leqslant
\end{gathered}
$$




$$
\begin{aligned}
\leqslant \frac{L_{0}}{2}\left(4\left\|x_{k}-x_{0}\right\|+\left\|y_{k}-x_{k}\right\|+\|\right. & \left.x_{0}-y_{0} \|\right) \leqslant \\
\leqslant \frac{2 L_{0}}{2}\left(4 t_{k}+s_{k}-t_{k}+s\right) & = \\
& =\frac{L_{0}}{2}\left(3 t_{k}+s_{k}+s\right)<1
\end{aligned}
$$

so

$$
\left\|A_{k}^{-1} A_{0}\right\| \leqslant \frac{1}{1-\frac{L_{0}}{2}\left(3 t_{k}+s_{k}+s\right)} .
$$

Using the method (2), we get the identity

$$
F\left(x_{k}\right)=F\left(x_{k}\right)-F\left(x_{k-1}\right)-\left[\frac{x_{k-1}+y_{k-1}}{2}, x_{k-1} ; F\right]\left(x_{k}-x_{k-1}\right)
$$

so, by (iii) and (27),

$$
\begin{gathered}
\left\|A_{0}^{-1} F\left(x_{k}\right)\right\| \leqslant L\left(\left\|x_{k}-\frac{x_{k-1}+y_{k-1}}{2}\right\|+\left\|x_{k}-x_{k-1}\right\|\right) \leqslant \\
\leqslant \frac{L}{2}\left\|2 x_{k}-\left(x_{k-1}+y_{k-1}\right)\right\|\left\|x_{k}-x_{k-1}\right\| \leqslant \\
\leqslant \frac{L}{2}\left(\left\|x_{k}-x_{k-1}\right\|+\left\|x_{k}-y_{k-1}\right\|\right)\left\|x_{k}-x_{k-1}\right\| \leqslant \\
\leqslant \frac{L}{2}\left(\left\|x_{k}-x_{k-1}\right\|+\left\|x_{k}-x_{k-1}\right\|+\left\|y_{k-1}-x_{k-1}\right\|\right)\left\|x_{k}-x_{k-1}\right\| \leqslant \\
\leqslant \frac{L}{2}\left(2\left(t_{k}-t_{k-1}\right)+\left(s_{k-1}-t_{k-1}\right)\right)\left(t_{k}-t_{k-1}\right) .
\end{gathered}
$$

Then, by (3), (25), (26) and (28) we obtain

$$
\begin{aligned}
\left\|y_{k}-x_{k}\right\|= & \left\|\left[B_{k}^{-1} A_{0}\right]\left[A_{0}^{-1} F\left(x_{k}\right)\right]\right\| \leqslant\left\|B_{k}^{-1} A_{0}\right\|\left\|A_{0}^{-1} F\left(x_{k}\right)\right\| \leqslant \\
& \leqslant \frac{L\left(2\left(t_{k}-t_{k-1}\right)+\left(s_{k-1}-t_{k-1}\right)\right)\left(t_{k}-t_{k-1}\right)}{2\left[1-\frac{L_{0}}{2}\left(t_{k-1}+s_{k-1}+2 t_{k}+s\right)\right]}=s_{k}-t_{k}
\end{aligned}
$$

and

$$
\begin{array}{r}
\left\|x_{k+1}-x_{k}\right\|=\left\|\left[A_{k}^{-1} A_{0}\right]\left[A_{0}^{-1} F\left(x_{k}\right)\right]\right\| \leqslant\left\|A_{k}^{-1} A_{0}\right\|\left\|A_{0}^{-1} F\left(x_{k}\right)\right\| \leqslant \\
\leqslant \frac{L\left(2\left(t_{k}-t_{k-1}\right)+\left(s_{k-1}-t_{k-1}\right)\right)\left(t_{k}-t_{k-1}\right)}{2\left[1-\frac{L_{0}}{2}\left(3 t_{k}+s_{k}+s\right)\right]}=t_{k+1}-t_{k}
\end{array}
$$

completing the induction for (22) and (23). We also have 


$$
\begin{aligned}
\left\|x_{k+1}-x_{0}\right\| \leqslant\left\|x_{k+1}-x_{k}\right\|+\ldots+\left\|x_{1}-x_{0}\right\| & \leqslant \\
& \leqslant t_{k+1}-t_{k}+\ldots+t_{1}-t_{0}=t_{k+1}<t_{*}
\end{aligned}
$$

and

$$
\left\|y_{k}-x_{0}\right\| \leqslant\left\|y_{k}-x_{k}\right\|+\left\|x_{k}-x_{0}\right\| \leqslant s_{k}-t_{k}+t_{k}-t_{0}=s_{k}<t_{*},
$$

so $y_{k}, x_{k+1} \in U\left(x_{0}, t_{*}\right)$. Moreover, the sequence $\left\{t_{k}\right\}$ is fundamental by Lemma 1. Hence, the sequence $\left\{x_{k}\right\}$ is fundamental too and, as such, it converges to some $x_{*} \in \bar{U}\left(x_{0}, t_{*}\right)$. By sending $k \rightarrow \infty$ in (28) and using the continuity of $F$, we conclude $F\left(x_{*}\right)=0$.

Concerning the uniqueness of the solution $x_{*}$, we have:

Proposition 1. Under the assumptions of Theorem 1, assume further that

$$
L_{0}\left(3 t_{*}^{1}+t_{*}\right)<2
$$

for some $t_{*}^{1} \geqslant t_{*}$. Then, $x_{*}$ is the only solution of the equation $F(x)=0$ in the set $\Omega_{1}=\Omega \cap U\left(x_{0}, t_{*}^{1}\right)$.

Proof. Let $x_{*}^{1} \in \Omega_{1}$ with $F\left(x_{*}^{1}\right)=0$. Set $T=\left[x_{*}, x_{*}^{1} ; F\right]$. Using (iii) and (31), we get

$$
\begin{aligned}
\left\|A_{0}^{-1}\left(T-A_{0}\right)\right\| & \leqslant L_{0}\left(\left\|x_{*}-\frac{x_{0}+y_{0}}{2}\right\|+\left\|x_{*}^{1}-x_{0}\right\|\right) \leqslant \\
& \leqslant L_{0}\left(\frac{\left\|x_{*}-x_{0}\right\|+\left\|x_{*}^{1}-x_{0}\right\|}{2}+\left\|x_{*}^{1}-x_{0}\right\|\right) \leqslant \\
& \leqslant L_{0}\left(\frac{t_{*}+t_{*}^{1}}{2}+t_{*}^{1}\right)<1,
\end{aligned}
$$

so $x_{*}=x_{*}^{1}$ is deduced, since $T$ is invertible and

$$
T\left(x_{*}-x_{*}^{1}\right)=F\left(x_{*}\right)-F\left(x_{*}^{1}\right)=0-0=0 .
$$

Remark. We can compute the computational order of convergence defined by

$$
a=\ln \left(\frac{\left\|x_{n+1}-x^{*}\right\|}{\left\|x_{n}-x^{*}\right\|}\right) / \ln \left(\frac{\left\|x_{n}-x^{*}\right\|}{\left\|x_{n-1}-x^{*}\right\|}\right)
$$

or the approximate computational order of convergence

$$
b=\ln \left(\frac{\left\|x_{n+1}-x_{n}\right\|}{\left\|x_{n}-x_{n-1}\right\|}\right) / \ln \left(\frac{\left\|x_{n}-x_{n-1}\right\|}{\left\|x_{n-1}-x_{n-2}\right\|}\right) .
$$


This way, we obtain, in practice, the order of convergence in a way that avoids high Fréchet derivatives for the operator $F$ and Taylor series used in other studies.

\section{Numerical Example.}

Let $B_{1}=B_{2}=\mathbb{R}^{3}, \Omega=U(0,1)$. Define $F$ on $\Omega$ by

$$
F(x)=F\left(u_{1}, u_{2}, u_{3}\right)=\left(e^{u_{1}}-1, \frac{e-1}{2} u_{2}^{2}+u_{2}, u_{3}\right)^{T} .
$$

For the points $u=\left(u_{1}, u_{2}, u_{3}\right)^{T}$, the Fréchet derivative is given by

$$
F^{\prime}(u)=\left(\begin{array}{ccc}
e^{u_{1}} & 0 & 0 \\
0 & (e-1) u_{2}+1 & 0 \\
0 & 0 & 1
\end{array}\right)
$$

Using the norm of the maximum of the rows for $x_{0}=\left(10^{-3}, 10^{-3}, 10^{-3}\right)^{T}$, $y=\left(10^{-4}, 10^{-4}, 10^{-4}\right)^{T}$, we get $L_{0}=0.7(e-1), L=e^{\rho}$, where $\rho=0.4118$. Then we have $s=0.0156, \eta=0.0015$,

$$
\gamma_{0}=0.0035<\gamma=0.6245<1-\frac{L_{0} \eta}{1-\frac{L_{0}}{2} s}=0.9985, \quad t_{* *}=0.0015 .
$$

We have verified all the conditions of Theorem 1 . Hence, we conclude that $\lim _{n \rightarrow \infty} x_{n}=x_{*}=(0,0,0)^{T}$.

\section{References}

[1] Appell J. De., Pascale E., Evkhuta N. A., Zabrejko P. P., On the two step Newton method for the solution of nonlinear equations. Math. Nachr., 1995, vol. 172 , pp. $5-14$.

[2] Argyros I. K. Computational Theory of Iterative Methods, Series: Studies in Computational Mathematics 15 . Editors: Chui C.K. and Wuytack L. Elsevier Publ. Company, New York, 2007.

[3] Argyros I. K., George S. Mathematical modeling for the solution of equations and systems of equations with applications. Volume-IV, Nova Publishes, NY, 2020.

[4] Ezquerro J. A., Hernandez M. A. An improvement of the region of accessibility of Chebyshev's method from Newton's method. Math. Comput., 2009, vol. 78 , pp. $1613-1627$.

DOI: https://doi.org/10.1090/S0025-5718-09-02193-0 
[5] Ezquerro J. A., Hernandez M. A., Magrenan A. A. Stating points for Newton's method under a center Lipschitz condition for the second derivative. J. Comput. Appl. Math., 2018, vol. 330, pp. 721-731.

DOI: https://doi.org/10.1016/j.cam.2016.12.013

[6] Kantorovich L. V., Akilov G. P. Functional Analysis. Oxford: Pergamon, 1982.

[7] Magreñán A. A., Argyros I. K. Two-step Newton methods. Journal of Complexity, 2014, vol. 30(4), pp. 533-553.

DOI: https://doi.org/10.1016/j.jco.2013.10.002

[8] Potra F. A, Ptak V. Nondiscrete induction and iterative processes, Research notes in Mathematics, 103, Pitman Boston, M.A, 1984.

Received October 8, 2020.

In revised form, October 13, 2020.

Accepted April 09, 2021.

Published online April 19, 2021.

Gus Argyros ${ }^{a}$

E-mail: Gus.Argyros@cameron.edu

Michael Argyros ${ }^{a}$

E-mail: Michael.Argyros@cameron.edu

Ioannis K. Argyrosv ${ }^{a}$

E-mail: iargyros@cameron.edu

Santhosh George ${ }^{b}$

E-mail: sgeorge@nitk.edu.in

a. Cameron University 2800 W. Gore Blvd. Lawton, OK 73505-6377, USA

b. National Institute of Technology

Karnataka, India-575 025 